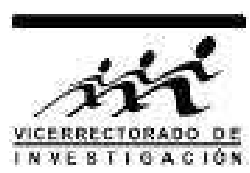

\title{
Propiedades ópticas de los calcogenuros de metales de transición 3D,2-2D y 2D
}

\author{
G. A. Valdivia, R. A. Montalvo y P. H. Rivera* \\ Facultad de Ciencias Físicas, Universidad Nacional Mayor de San Marcos, Lima, Perú
}

Recibido 20 diciembre 2015 - Aceptado 22 enero 2016

\begin{abstract}
En el presente trabajo realizamos un estudio sistemático de las propiedades ópticas de los dicalcogenuros de metales de transición usando la teoría de funcionales de densidad dependiente del tiempo para determinar los estados excitados de la banda de conducción considerando que la densidad electrónica es una funcional que depende del tiempo y afecta temporalmente el potencial coulombiano de apantallamiento que se usa en la ecuación de Dyson para determinar la susceptibilidad eléctrica. Calculamos las partes reales e imaginarias de la función dieléctrica que nos permite observar la absorción y la formación de plasmones para fotones de la radiación electromagnética que abarca desde el infrarrojo $(25 \mathrm{meV})$ hasta el ultravioleta $(30 \mathrm{eV})$. $\mathrm{Y}$ encontramos que las partes imaginarias de las componentes $z z$ de los sistemas $2 \mathrm{D}$ no presentan picos de absorción, mientras que, las componentes $x x, y y$ y $z z$ de los sistemas $2 \mathrm{D}$ no presentan ninguna frecuencia de plasmones significativos.
\end{abstract}

Palabras claves: Función dieléctrica, absorción, frecuencia de plasma, dicalcogenuros.

\section{Optical properties of the 3D, 2-2D and 2D transition metal dichal- cogenides}

In the present work we realize a systematic study of the optical properties of transition metal dichalcogenides using time dependent density functional theory for the determination of excited states of conduction band considering the electron density as a time dependent functional and temporarily affect the screening Coulomb potential which is used in Dyson equation for electric susceptibility determination.

We calculate the real and imaginary parts of dielectric function which permit us to observe the absorption coefficient and the plasmon formation for electromagnetic radiation photons from the infrared $(25 \mathrm{meV})$ to ultraviolet $(30 \mathrm{eV})$ range. And we found that the imaginary parts of $z z$ component of the $2 \mathrm{D}$ systems have not absorption peaks, meanwhile, the $x x, y y$ and $z z$ components of the $2 \mathrm{D}$ systems have not shown plasma frequencies.

Keywords: Dielectric function, absorption, plasma frequencies, dichalcogenides.

La interacción de un campo electromagnético con la estructura electrónica de un determinado material es un proceso que observamos constantemente en la naturaleza. La comprensión de los detalles que involucra este proceso está aún en abierto para una multitud de materiales cuyos espectros de absorción obtenidos experimentalmente son diferentes a los obtenidos teóricamente [1]. Para el diseño de nuevos dispositivos optoelectrónicos

*priverar@unmsm.edu.pe 
usando nuevos materiales para ser usados en la industria y la medicina es imprescindible tener modelos numéricos que detalladamente muestren un espectro de absorción con características requeridas para determinadas aplicaciones que no tengan un impacto negativo al medio ambiente y a la salud humana.

En los últimos años, una alternativa para los modelos que usan la aproximación GW [2] y la ecuación de BetheSalpeter [1] ha sido la teoría de funcionales de densidad dependientes del tiempo, Time Dependent Density Functional Theory, TDDFT [3, 4], este modelo usa la funcional de densidad dependiente del tiempo que se incorpora mediante la dependencia temporal del potencial externo aplicado al sistema que induce una polarización como respuesta lineal del sistema y simultáneamente provoca un apantallamiento de los portadores de carga cuyas posiciones dinámicamente varían con el tiempo, el efecto es que el potencial de intercambio y correlación que es una funcional que depende de la densidad, en el espacio recíproco, depende de la frecuencia [5].

Con este enfoque se calcula los estados excitados, el espectro de absorción y la función de pérdida de energía de los electrones que permiten analizar las propiedades ópticas de los calcogenuros de metales de transición [7] estudiando sistemáticamente en sus fases cristalinas tridimensionales o bulk, bicapas y como sistemas cristalinos bidimensionales.

Este trabajo comprende un breve análisis teórico del modelo, seguido por la discusión de los resultados numéricos obtenidos, luego exponemos las conclusiones del trabajo y las referencias.

\section{Teoría}

Cuando un campo electromagnético externo $\mathcal{E}(\boldsymbol{r}, t)=\mathcal{E}(q, \omega) \cos (\boldsymbol{q} \cdot \boldsymbol{r}-\omega t)$ es aplicado sobre un sistema dieléctrico mesoscópico que se encuentra en el vacío, fenomenológicamente y de acuerdo a la teoría de respuesta lineal, la polarización inducida en una dirección $i$ es definida como $P_{i}(\boldsymbol{r}, t)=$ $\epsilon_{0} \sum_{j} \chi_{i j}\left(\boldsymbol{r}, t ; \boldsymbol{r}^{\prime}, t^{\prime}\right) \mathcal{E}_{j}\left(\boldsymbol{r}^{\prime}, t^{\prime}\right)$, donde $\chi_{i j}$ es un tensor de segundo rango conocido como tensor de susceptibilidad eléctrica y $\epsilon_{0}$ es la constante eléctrica. Considerando que los valores esperados del operador de evolución y el de traslación son homogéneos, -puesto que las perturbaciones dependientes del tiempo y las correcciones locales de campo son evitadas por el valor esperado de dichos operadores evaluados en una celda unitaria-, usamos el teorema de la convolución y la transformada de Fourier de manera tal que la polarización se expre- sa como $P_{i}(\boldsymbol{q}, \omega)=\epsilon_{0} \sum_{j} \chi_{i j}(\boldsymbol{q}, \omega) \mathcal{E}_{j}(\boldsymbol{q}, \omega)$. Se debe tener en cuenta que, mientras $\mathcal{E}(\boldsymbol{r}, t), \boldsymbol{P}(\boldsymbol{r}, t)$ y la susceptibilidad $\chi_{i j}\left(\boldsymbol{r}, t ; \boldsymbol{r}^{\prime}, t^{\prime}\right)$ son reales, sus respectivas transformadas de Fourier son complejas [6, 8].

Por otro lado, la componente $i$ del campo de desplazamiento eléctrico $D_{i}(\boldsymbol{r}, t)=\epsilon_{0} \mathcal{E}_{i}(\boldsymbol{r}, t)+P_{i}(\boldsymbol{r}, t)$ $=\epsilon_{0} \mathcal{E}_{i}(\boldsymbol{r}, t)+\epsilon_{0} \sum_{j} \chi_{i j}\left(\boldsymbol{r}, t ; \boldsymbol{r}^{\prime}, t^{\prime}\right) \mathcal{E}_{j}\left(\boldsymbol{r}^{\prime}, t^{\prime}\right)$, mientras que su transformada de Fourier $D_{i}(\boldsymbol{q}, \omega)=$ $\epsilon_{0} \varepsilon_{i j}(\boldsymbol{q}, \omega) \mathcal{E}_{i}(\boldsymbol{q}, \omega)$, donde $\varepsilon_{i j}(\boldsymbol{q}, \omega)$ es el tensor dieléctrico, también de segundo rango [6, 8, 9].

La relación bastante conocida que existe entre el tensor de susceptibilidad eléctrica y el tensor dieléctrico se describe como

$$
\varepsilon_{i j}(\boldsymbol{q}, \omega)=1+\chi_{i j}(\boldsymbol{q}, \omega),
$$

denotando como $\varepsilon_{r}(\boldsymbol{q}, \omega)$ y $\varepsilon_{i}(\boldsymbol{q}, \omega)$, la parte real e imaginaria del tensor dieléctrico. Cabe recordar que en este contexto el tensor dieléctrico cumple que $\varepsilon(-\boldsymbol{q},-\omega)=$ $\varepsilon^{*}(\boldsymbol{q}, \omega)$ y $\varepsilon_{i j}(\boldsymbol{q}, \omega)=\varepsilon_{j i}(-\boldsymbol{q}, \omega)$, conocidos como las relaciones de Onsager [6, 8].

Experimentalmente, se mide la reflectancia $\mathcal{R}$ de manera perpendicular o con un ángulo de incidencia $\phi$ respecto a la normal mediante elipsómetros. En el primer caso, la reflectancia se relaciona con el índice de refracción complejo $\tilde{n}$ a través de la relación

$$
\tilde{n}=\frac{1+\mathcal{R}^{2}}{1-\mathcal{R}^{2}},
$$

y en el segundo, mediante las relaciones de Fresnel

$$
\begin{aligned}
& \mathcal{R}_{\mathrm{s}}=\left|r_{\mathrm{s}}\right|^{2}=\left|\frac{\cos \phi-\sqrt{\tilde{n}^{2}-\operatorname{sen}^{2} \phi}}{\cos \phi+\sqrt{\tilde{n}^{2}-\operatorname{sen}^{2} \phi}}\right|^{2} \mathrm{y} \\
& \mathcal{R}_{\mathrm{p}}=\left|r_{\mathrm{p}}\right|^{2}=\left|\frac{\tilde{n}^{2} \cos \phi-\sqrt{\tilde{n}^{2}-\operatorname{sen}^{2} \phi}}{\tilde{n}^{2} \cos \phi+\sqrt{\tilde{n}^{2}-\operatorname{sen}^{2} \phi}}\right|^{2},
\end{aligned}
$$

donde $\mathcal{R}_{\mathrm{s}}\left(r_{\mathrm{s}}\right)$ y $\mathcal{R}_{\mathrm{p}}\left(r_{\mathrm{p}}\right)$ son las reflectancias (reflectividades complejas) producidas por las componentes polarizadas s (vertical) y $\mathrm{p}$ (paralela) del haz incidente en el plano de incidencia [6, 10].

En el índice de refracción complejo $\tilde{n}=n+i \varkappa$, la parte real $n$ expresa el índice de refracción propiamente dicho del medio dieléctrico y la parte imaginaria $\varkappa$ es el coeficiente o índice de extinción. Cuando un haz de radiación electromagnética incide sobre un sistema dieléctrico, la intensidad $I$ de la radiación disminuye entre dos puntos $\boldsymbol{r}_{1}$ y $\boldsymbol{r}_{2}$ dentro del medio mediante la ecuación $I\left(\boldsymbol{r}_{2}\right)=I\left(\boldsymbol{r}_{1}\right) \exp \left[-\alpha\left|\boldsymbol{r}_{2}-\boldsymbol{r}_{1}\right|\right]$ donde $\alpha$ es el coeficiente de absorción, que a su vez está relacionado 
con el coeficiente de extinción como

$$
\alpha=\frac{4 \pi \varkappa}{\lambda_{0}}
$$

donde $\lambda_{0}$ es la longitud de onda del campo electromagnético en el vacío, esto implica que el índice de refracción complejo depende del coeficiente de absorción; si los fotones de la radiación electromagnética tienen una energía mayor que la energía de los fonones ópticos y acústicos del material dieléctrico y menor que la energía del band-gap, el coeficiente de absorción es aproximadamente cero, esto implica que el índice de refracción es un número real puro. Obviamente si la energía de los fotones es mayor que la energía del band-gap, el coeficiente de absorción crece muy rápidamente de modo que la profundidad de penetración óptica $\alpha^{-1}$ se hace pequeño de modo que las muestras deben ser cada vez más delgadas para medir $\alpha$ en un rango razonable de energía de los fotones [6].

Por ese motivo, de las mediciones experimentales de las reflectancias (reflectividades) $\mathcal{R}_{\mathrm{s}}\left(r_{\mathrm{s}}\right), \mathcal{R}_{\mathrm{p}}\left(r_{\mathrm{p}}\right)$ a un ángulo de incidencia $\phi$ fijo y del coeficiente de absorción $\alpha$ del medio dieléctrico se determina el índice de refracción complejo $\tilde{n}$ que está relacionado a la función dieléctrica como [6]

$$
\varepsilon=\operatorname{sen}^{2} \phi+\operatorname{sen}^{2} \phi \tan ^{2} \phi\left(\frac{1-\sigma}{1+\sigma}\right)^{2},
$$

donde $\sigma=r_{\mathrm{p}} / r_{\mathrm{s}}$, la razón entre las reflectividades complejas, y para una incidencia perpendicular como

$$
\varepsilon(\omega)=(\tilde{n})^{2} .
$$

Desde el punto de vista teórico, en el marco de la teoría de respuesta lineal, calculamos la función dieléctrica como

$$
\varepsilon(\boldsymbol{q}, \omega)=1-v(\boldsymbol{q}) \chi(\boldsymbol{q}, \omega),
$$

donde $v$ es el potencial de apantallamiento inducido por el campo electromagnético externo y la susceptibilidad eléctrica $\chi$ se obtiene vía la ecuación de Dyson [11],

$$
\chi=\chi_{0}+\chi_{0}\left[v+f_{\mathrm{xc}}^{(2)}\right] \chi,
$$

donde $\chi_{0}$ es la susceptibilidad eléctrica de Kohn-Sham, mientras que $f_{\mathrm{xc}}^{(2)}$ es el kernel de dos puntos para la interacción de intercambio y correlación dentro del esquema de la teoría de funcionales de densidad dependiente del tiempo, TDDFT [3].
Haciendo una breve digresión, mencionamos que una cantidad muy útil en el estudio de las propiedades ópticas de los materiales y directamente observable en la espectroscopia de pérdida de energía de los electrones, Electron Energy Loss Spectrocopy, EELS, es la denominada función de pérdida que se define como [6,12]

$$
L(\boldsymbol{q}, \omega)=-\operatorname{Im} \frac{1}{\varepsilon(\boldsymbol{q}, \omega)} .
$$

La susceptibilidad eléctrica de Kohn-Sham es calculada mediante la expresión [4],

$$
\begin{gathered}
\chi_{0}(\boldsymbol{q}, \omega)=\frac{1}{V} \sum_{n, m, \boldsymbol{k}} \frac{f_{n, \boldsymbol{k}}-f_{m, \boldsymbol{k}+\boldsymbol{q}}}{E_{n, \boldsymbol{k}}-E_{m, \boldsymbol{k}+\boldsymbol{q}}+\hbar \omega+i \delta} \times \\
M_{n m \boldsymbol{k}}(\boldsymbol{q}, \boldsymbol{G}) M_{n m \boldsymbol{k}}^{*}\left(\boldsymbol{q}, \boldsymbol{G}^{\prime}\right),
\end{gathered}
$$

donde $f_{l, \boldsymbol{z}}$ son los números de ocupación que están dados por la distribución de Fermi-Dirac a temperatura cero; $m$ y $n$ son los autoestados que incluyen los grados de libertad de los espínes; $E_{l, \boldsymbol{z}}$ son los autovalores de energía para los autoestados de partícula simple $|l, \boldsymbol{z}\rangle$ obtenidos mediante TDDFT; $\omega$ es la frecuencia del campo electromagnético y $\delta$ es un número infinitesimal en energía; mientras que las matrices $M_{n m \boldsymbol{k}}$ son definidas como [4],

$$
M_{n m \boldsymbol{k}}(\mathbf{q}, \mathbf{G})=\left\langle E_{n}, \boldsymbol{k}\left|e^{-i(\boldsymbol{q}+\boldsymbol{G}) \cdot \boldsymbol{r}}\right| E_{m}, \boldsymbol{k}+\boldsymbol{q}\right\rangle .
$$

En el contexto de la TDDFT, la evolución de las autofunciones de onda obedecen la ecuación de Schrödinger descrita por Kohn y Sham como

$$
i \hbar \frac{\partial}{\partial t} \phi_{j}(\boldsymbol{r}, t)=\left[-\frac{\hbar^{2}}{2 m} \nabla^{2}+v_{\text {eff }}[n](\boldsymbol{r}, t)\right] \phi_{j}(\boldsymbol{r}, t)
$$

donde $\phi_{j}(\boldsymbol{r}, t)=\left\langle\boldsymbol{r}(t) \mid E_{j}\right\rangle$ son los orbitales de KohnSham de modo que la densidad es expresada como [13]

$$
n(\boldsymbol{r}, t)=\sum_{j} \Theta\left(\mu-E_{j}\right)\left|\left\langle\boldsymbol{r}(t) \mid E_{j}\right\rangle\right|^{2}
$$

considerando $\mu$ como el potencial químico que es el máximo valor que toman los autovalores de energía $E_{j}$ de los estados ocupados $\left|E_{j}\right\rangle$ en la banda de valencia. Usando el teorema de Runge-Gross [3], el potencial efectivo $v_{\text {eff }}$ es una funcional dependiente de la densidad $n(\boldsymbol{r}, t)$ descrita como

$$
\begin{aligned}
& v_{\text {eff }}[n](\boldsymbol{r}, t)=v_{\text {ext }}(\boldsymbol{r}, t)+\int d \boldsymbol{r}^{\prime} \frac{n\left(\boldsymbol{r}^{\prime}, t\right)}{\left|\boldsymbol{r}-\boldsymbol{r}^{\prime}\right|}+ \\
& v_{\text {xc }}[n](\boldsymbol{r}, t) \text {, }
\end{aligned}
$$


observando que entre los tres términos únicamente el potencial de intercambio y correlación $v_{x c}$ es una funcional que depende de la densidad, mientras que el potencial debido a los campos electromagnéticos externos $v_{\text {ext }}$ origina la dependencia temporal de la respuesta lineal del sistema induciendo la polarización y el apantallamiento simultáneo de los portadores de carga [4].

\section{Procedimiento}

El método propuesto por el TDDFT [3] ha sido implementado en el Exciting [14, 15]. El cual es un conjunto de programas que calculan mediante el DFT la estructura electrónica de un material hasta la banda de valencia usando la aproximación LDA (local density approximation) [17] e implementa el cálculo dependiente del tiempo de los estados excitados de un sistema de muchos cuerpos como alternativa a los métodos perturbativos de muchos cuerpos [11], este cálculo se realiza usando el método de la aproximación de fase aleatoria Random Phase Approximation, RPA-, que es equivalente a la regla de oro de Fermi para los modelos de partícula simple [16].

La estructura cristalina de los dicalcogenuros de los metales de transición tales como $\mathrm{MoS}_{2}, \mathrm{MoSe}_{2}, \mathrm{WS}_{2}$ y $\mathrm{WSe}_{2}$ son trigonales prismáticas conformadas por dos capas de calcogenuros ( $\mathrm{S} \circ \mathrm{Se}$ ) y una capa intermedia de metales de transición (Mo o W) con un corrimiento espacial tal como se muestra en la Figura 1 , de modo tal que, observándose desde arriba en la dirección $z$ se observa una red hexagonal parecida al grafeno de los átomos proyectados en el plano $x y$.

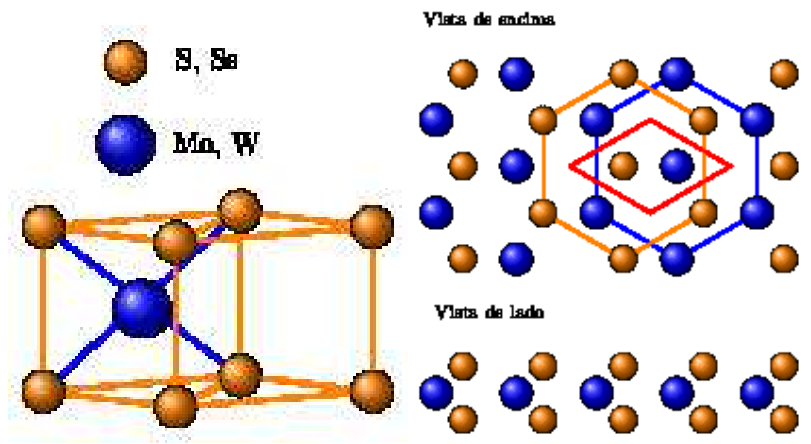

Figura 1: Se muestra la estructura trigonal prismática de los calcogenuros ( $\mathrm{S}$ o $\mathrm{Se}$ ) de los metales de transición (Mo o W) que pertenece al grupo espacial $\mathrm{P}_{3} / \mathrm{mmc}$.

Asimismo hemos analizado las propiedades ópticas numéricamente de $\mathrm{MoS}_{2}, \mathrm{MoSe}_{2}, \mathrm{WS}_{2}$ y $\mathrm{WSe}_{2}$ en sus formas bulk 3D, bicapa 2-2D y monocapa 2D. Cabe mencionar que estamos considerando una monocapa al arreglo de las dos capas de dicalcogenuros más la capa intermediaria de metales de transición. La red unitaria, las formas 2D, 2-2D y 3D son mostradas en la Figura 2.

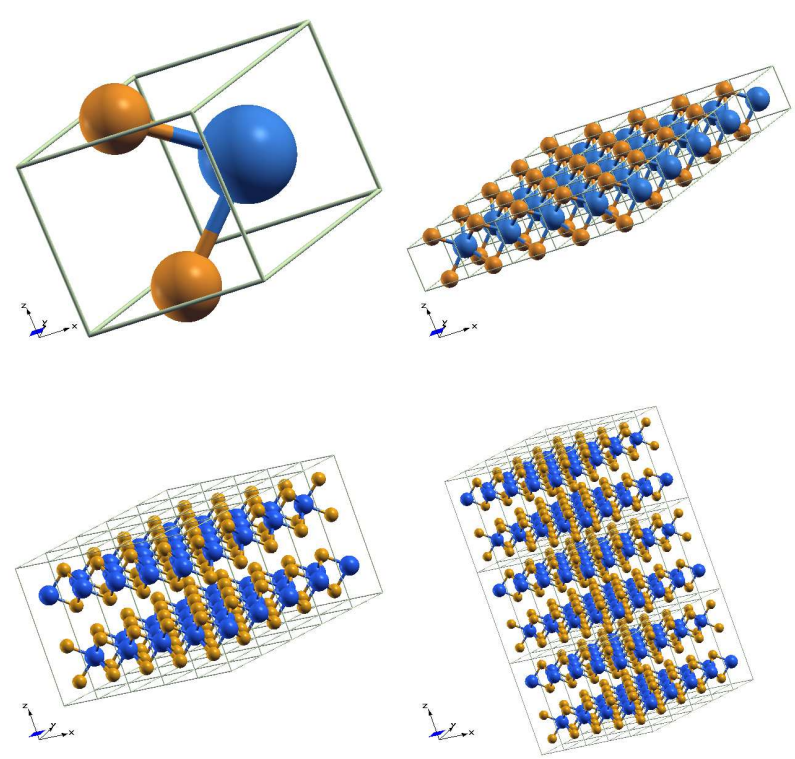

Figura 2: Se muestran, de izquierda a derecha y de arriba hacia abajo, la celda unitaria, el arreglo 2D, el arreglo 2-2D y el arreglo 3D para los dicalcogenuros de los metales de transición.

Los parámetros de red para los diferentes dicalcogenuros de metales de transición utilizados en el presente trabajo están resumidos en la tabla 1.

\begin{tabular}{lcccc}
\hline $\begin{array}{l}\text { Parámetro } \\
\text { de red }(\AA)\end{array}$ & $\mathrm{MoS}_{2}$ & $\mathrm{MoSe}_{2}$ & $\mathrm{WS}_{2}$ & $\mathrm{WSe}_{2}$ \\
\hline $\mathrm{a}$ & 3.122 & 3.288 & 3.147 & 3.297 \\
$\mathrm{c}$ & 11.986 & 12.931 & 12.167 & 12.982 \\
\hline
\end{tabular}

Tabla 1: Parámetro de red de los dicalcogenuros de metales de transición.

\section{Resultados}

A partir de la Figura 3 hasta la Figura 10 se muestran los resultados obtenidos de las partes reales e imaginarias de las componentes $x x$ y $z z$ de las funciones dieléctricas graficadas simultáneamente con las funciones de pérdida de energía de los electrones con respecto a la energía de los fotones de la radiación electromagnética incidente en las muestras. Observamos que las componentes reales de 
las componentes $x x$ y $z z$ de la función dieléctricas están asociadas a la parte real de las componentes $x x$ y $z z$ de la parte real del índice de refracción complejo, mientras que las componentes imaginarias $x x$ y $z z$ de la función dieléctrica están asociados al coeficiente de absorción. Cabe mencionar que la componente $x x$ e $y y$ son aproximadamente iguales en todos los sistemas estudiados, las diferencias encontradas son mínimas, por ello sólo hemos considerado la componente $x x$ en todas las figuras correspondientes a todos los dicalcogenuros considerados $\mathrm{MoS}_{2}, \mathrm{MoSe}_{2}, \mathrm{WS}_{2}$ y WSe $\mathrm{W}_{2}$ en el estudio.

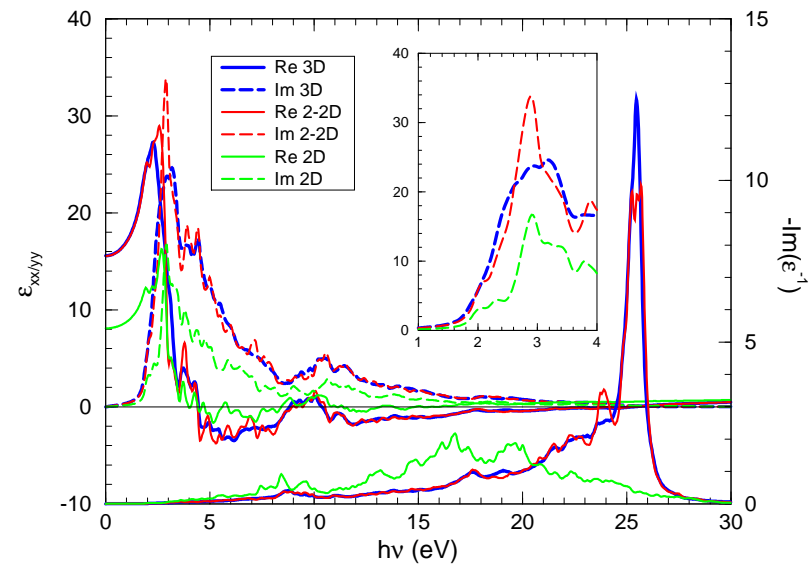

Figura 3: Se muestran los espectros de las componentes reales (líneas continuas) e imaginarias (líneas discontinuas) de la función dieléctrica $\varepsilon_{x x / y y}$ y la función de pérdida $\operatorname{Im}\left(\varepsilon^{-1}\right)$ (líneas continuas) con respecto a la energía de los fotones que abarca desde el infrarrojo hasta el ultravioleta para la molibdenita, $\mathrm{MoS}_{2}$, en sus formas 2D (verde), bicapas (rojo) y $3 \mathrm{D}$ (azul). En el recuadro se muestra una ampliación de $\varepsilon_{i}$ asociado a la absorción.

Para todos estos materiales las componentes reales $x x$ e yy de la función dieléctrica son mayores respecto a las componentes reales $z z$ en un barrido de energía de los fotones que va desde el infrarrojo $\approx 0.025$ $\mathrm{eV}(\lambda=495936.75 \AA)$ hasta el ultravioleta $\approx 30 \mathrm{eV}$ $(\lambda=413.28 \AA)$.

En todos los casos las componentes reales e imaginarias $x x$ y $z z$ de las funciones diélectricas para la forma 2D son totalmente diferentes de las formas 2-2D y 3D, en estas dos últimas sus funciones dieléctricas son casi parecidas con pocas excepciones en intensidad para determinadas energías.

Analizando las formas 3D de los $\mathrm{MoS}_{2}, \mathrm{MoSe}_{2}, \mathrm{WS}_{2}$ y $\mathrm{WSe}_{2}$, observamos que las componentes reales $x x$ de la función dieléctrica alcanza picos máximos de $\approx 27.27$ y 27.13 para $\mathrm{MoS}_{2}$ y $\mathrm{MoSe}_{2}$ a las energías de 2.279 y
$1.562 \mathrm{eV}$, respectivamente. Mientras que para $\mathrm{WS}_{2}$ y $\mathrm{WSe}_{2}$ los picos máximos son 23.68 y 23.85 a las energías de 2.375 y $1.553 \mathrm{eV}$, respectivamente. Se observa que para los materiales con Mo los picos de la componente real $x x$ de la función dieléctrica son mayores respecto a los materiales con W. Esto significa que conforme el átomo de transición se hace más pesado, atómicamente, el correspondiente pico máximo de la componente real $x x$ de la función dieléctrica disminuye. Luego el comportamiento de las curvas disminuyen alcanzando mínimos negativos entre 4 y $6 \mathrm{eV}$ y luego todos evolucionan hasta que alcanzan cero en torno de $\approx 23-25 \mathrm{eV}$.

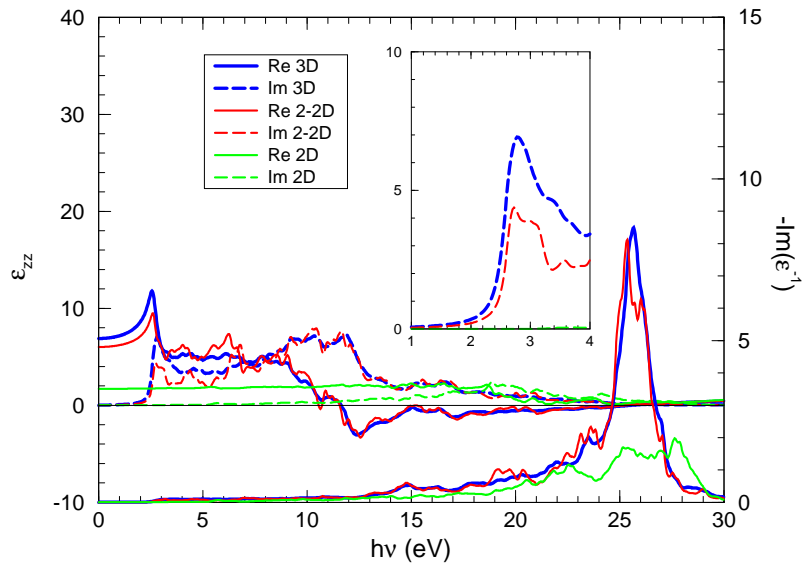

Figura 4: Se muestran los espectros de las componentes reales (líneas continuas) e imaginarias (líneas discontinuas) de la función dieléctrica $\varepsilon_{z z}$ y la función de pérdida $\operatorname{Im}\left(\varepsilon^{-1}\right)$ (líneas continuas) con respecto a la energía de los fotones que abarca desde el infrarrojo hasta el ultravioleta para la molibdenita, $\mathrm{MoS}_{2}$, en sus formas 2D (verde), bicapas (rojo) y $3 \mathrm{D}$ (azul). En el recuadro se muestra una ampliación de $\varepsilon_{i}$ asociado a la absorción.

Los metales de transición como el Mo y el W tienen los electrones $5 s^{1}-4 d^{5}$ y $6 s^{2}-4 f^{14}-5 d^{4}$, respectivamente, como las capas más externas. Obviamente el W es más pesado atómicamente que el Mo, por tanto, esto parece influir en la intensidad de los picos de la componente real $x x$ de la función dieléctrica puesto que los picos que corresponden al Mo son más intensos que los del $W$ y respecto a la presencia de los átomos calcogenuros como el $\mathrm{S}$ y el Se, cuyos electrones más externos son $3 s^{2}-3 p^{4}$ y $4 s^{2}-3 d^{10}-4 p^{4}$, respectivamente; siendo el Se más pesado que el $\mathrm{S}$, el efecto sobre los picos de la función dieléctrica es que cuanto más pesado sea el calcogenuro la posición de los picos se produce a menor energía de los fotones.

Observando la componente real $z z$ de la función die- 
léctrica para las formas 3D de los $\mathrm{MoS}_{2}, \mathrm{MoSe}_{2}, \mathrm{WS}_{2}$ y $\mathrm{WSe}_{2}$, los picos máximos ocurren con diferentes intensidades para cada caso. Sólo se puede comparar con respecto al átomo calcogenuro, los picos ocurren a menores energías de los fotones cuando el calcogenuro es más pesado, esto es congruente al comportamiento de los picos de la componente $x x$ mencionado en el párrafo anterior.

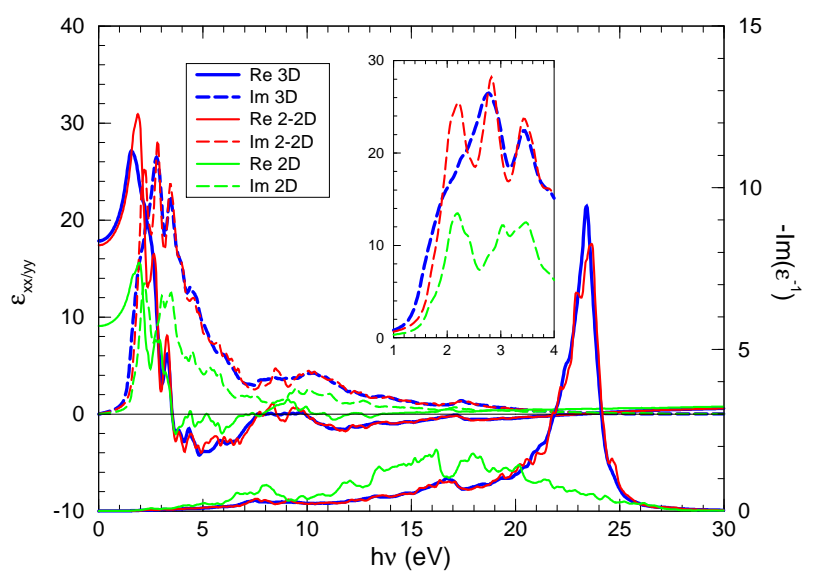

Figura 5: Se muestran los espectros de las componentes reales (líneas continuas) e imaginarias (líneas discontinuas) de la función dieléctrica $\varepsilon_{x x / y y}$ y la función de pérdida $\operatorname{Im}\left(\varepsilon^{-1}\right)$ (líneas continuas) con respecto a la energía de los fotones que abarca desde el infrarrojo hasta el ultravioleta para el $\mathrm{MoSe}_{2}$, en sus formas 2D (verde), bicapas (rojo) y 3D (azul). En el recuadro se muestra una ampliación de $\varepsilon_{i}$ asociado a la absorción.

Ahora analizamos la componente imaginaria de la función dieléctrica para los $\mathrm{MoS}_{2}, \mathrm{MoSe}_{2}, \mathrm{WS}_{2}$ y $\mathrm{WSe}_{2}$, que está asociada a los coeficientes de absorción. Comparando las componentes $x x$ y $z z$ se aprecia que los picos de absorción mejor definidos son las que corresponde a la componente $z z$ que se muestran para las energías de $2.776,2.151,2.751$ y $2.301 \mathrm{eV}$ para los respectivos materiales mencionados arriba, Figura 4, 6, 8 y 10, respectivamente. Observándose que para los calcogenuros más pesados la energía de los excitones es de menor energía, en este caso $2.151 \mathrm{eV}$ para $\mathrm{MoSe}_{2}$, Figura [6 y 2.301 eV para $\mathrm{WSe}_{2}$, Figura 10 que corresponde físicamente a que el Se posee mayor carga nuclear efectiva que permite una mayor localización de los portadores de carga, en cambio, para el $\mathrm{S}$ los portadores de carga están más extendidos o delocalizados porque la influencia de la carga nuclear efectiva es menor en la posición de los portadores de carga.

En seguida, hacemos un análisis de las componentes imaginarias $x x$ y $z z$ de las funciones dieléctricas para: 1$)$ $\mathrm{MoS}_{2}$, 2) $\mathrm{MoSe}_{2}$, 3) $\mathrm{WS}_{2}$ y 4) $\mathrm{WSe}_{2}$. En el primer caso, Figura 3 y 4 se observa que la componente $x x$ de la forma 3D presenta un perfil de absorción que contendría tres picos superpuestos ubicados en 2.59, 2.92 y 3.17 $\mathrm{eV}$, alcanzando en este último el valor máximo de todo el perfil, Figura 3 Observando el perfil de absorción de la forma 2-2D observamos un perfil de absorción compuesto de tres pico superpuestos cuyo primer pico está en $\approx 2.10 \mathrm{eV}$, el segundo en $\approx 2.45 \mathrm{eV}$ y el tercero más intenso en $\approx 2.90 \mathrm{eV}$. El perfil de absorción que corresponde a la forma 2D se observa claramente dos picos de absorción de menor intensidad en 2.06 y $2.33 \mathrm{eV}$ y un tercero más intenso en $2.90 \mathrm{eV}$. Con respecto a la componente $z z$ se observa que no existe absorción para el caso $2 \mathrm{D}$, para el caso 2-2D se observa un pico a $2.73 \mathrm{eV}$, mientras para el caso 3D se observa el pico de absorción en $2.78 \mathrm{eV}$.

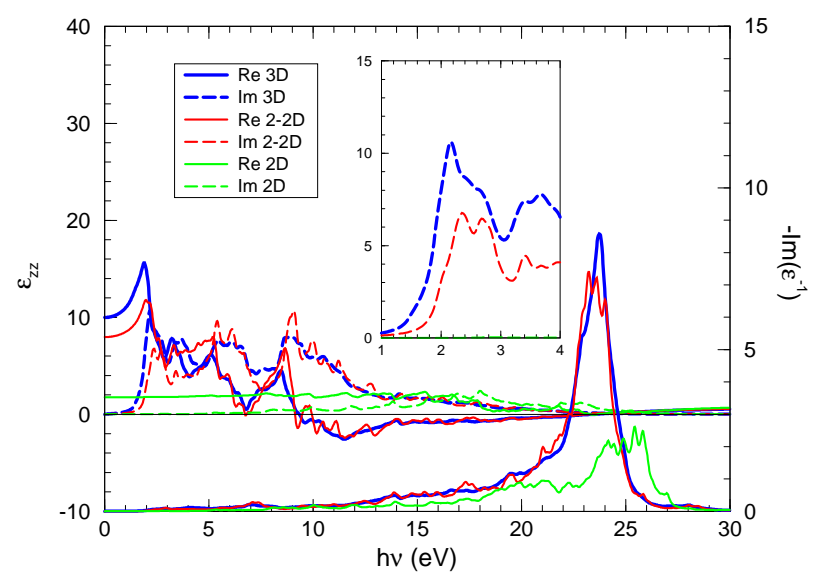

Figura 6: Se muestran los espectros de las componentes reales (líneas continuas) e imaginarias (líneas discontinuas) de la función dieléctrica $\varepsilon_{z z}$ y la función de pérdida $\operatorname{Im}\left(\varepsilon^{-1}\right)$ (líneas continuas) con respecto a la energía de los fotones que abarca desde el infrarrojo hasta el ultravioleta para el $\mathrm{MoSe}_{2}$, en sus formas 2D (verde), bicapas (rojo) y 3D (azul). En el recuadro se muestra una ampliación de $\varepsilon_{i}$ asociado a la absorción.

Para el segundo caso, Figura 5 y 6, la componente imaginaria $z z$ de la función dieléctrica del $\mathrm{MoSe}_{2}$ muestra para la forma 3D un perfil de absorción con pico definido en $2.15 \mathrm{eV}$, aunque el perfil de absorción en la componente $x x$ se muestran un pico máximo en $2.76 \mathrm{eV}$. Aqui cabe comentar que estos perfiles para la forma 3D parecen corresponder a dos picos de absorción que para $z z$ uno es máximo y para $x x$ el otro es máximo. Además existe un segundo perfil de absorción cuyo máximo está 
para $x x$ en $\approx 3.40 \mathrm{eV}$ y para $z z$ en $\approx 3.4 \mathrm{y} \approx 3.68 \mathrm{eV}$ correspondiendo a dos picos. Para la forma 2-2D la componente $x x$ presenta tres picos claramente diferenciados en $\approx 2.20,2.83$ y $3.43 \mathrm{eV}$, mientras que la componente $z z$ presenta tres picos para $\approx 2.35,2.68$ y $3.40 \mathrm{eV}$ teniendo el pico de mayor intensidad en la primera energía. Para la forma 2D, la componente $x x$ presenta tres picos de absorción en $\approx 2.20,3.03$ y $3.48 \mathrm{eV}$, la componente $z z$ no muestra espectro de absorción en la forma 2D.

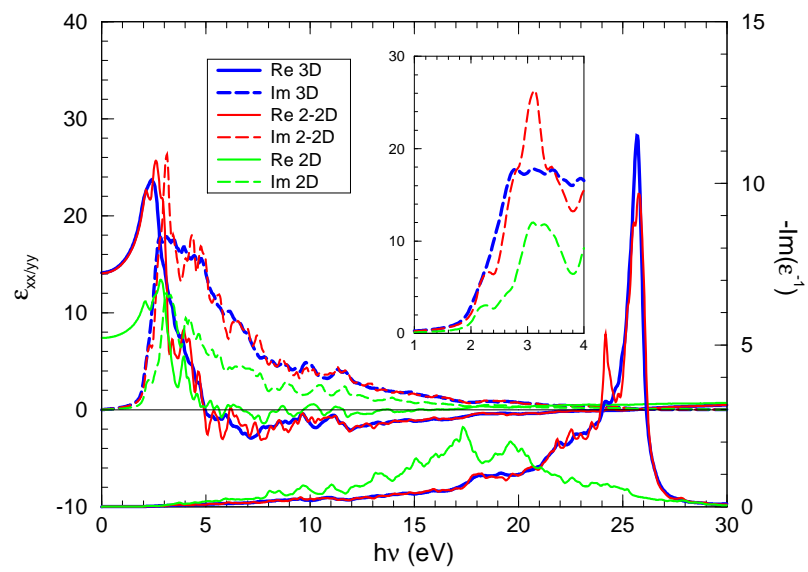

Figura 7: Se muestran los espectros de las componentes reales (líneas continuas) e imaginarias (líneas discontinuas) de la función dieléctrica $\varepsilon_{x x / y y}$ y la función de pérdida $\operatorname{Im}\left(\varepsilon^{-1}\right)$ (líneas continuas) con respecto a la energía de los fotones que abarca desde el infrarrojo hasta el ultravioleta para el $W_{2}$, en sus formas 2D (verde), bicapas (rojo) y 3D (azul). En el recuadro se muestra una ampliación de $\varepsilon_{i}$ asociado a la absorción.

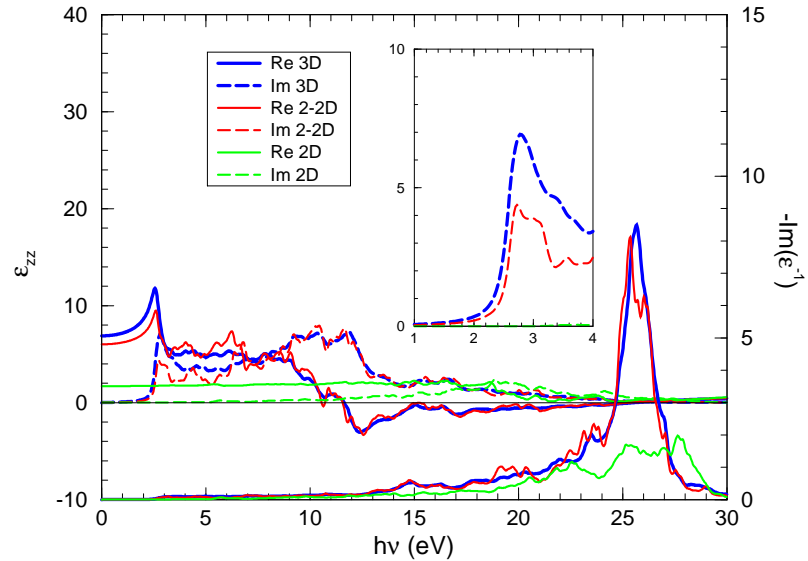

Figura 8: Se muestran los espectros de las componentes reales (líneas continuas) e imaginarias (líneas discontinuas) de la función dieléctrica $\varepsilon_{z z}$ y la función de pérdida $\operatorname{Im}\left(\varepsilon^{-1}\right)$ (líneas continuas) con respecto a la energía de los fotones que abarca desde el infrarrojo hasta el ultravioleta para el $W_{2}$, en sus formas 2D (verde), bicapas (rojo) y 3D (azul). En el recuadro se muestra una ampliación de $\varepsilon_{i}$ asociado a la absorción.

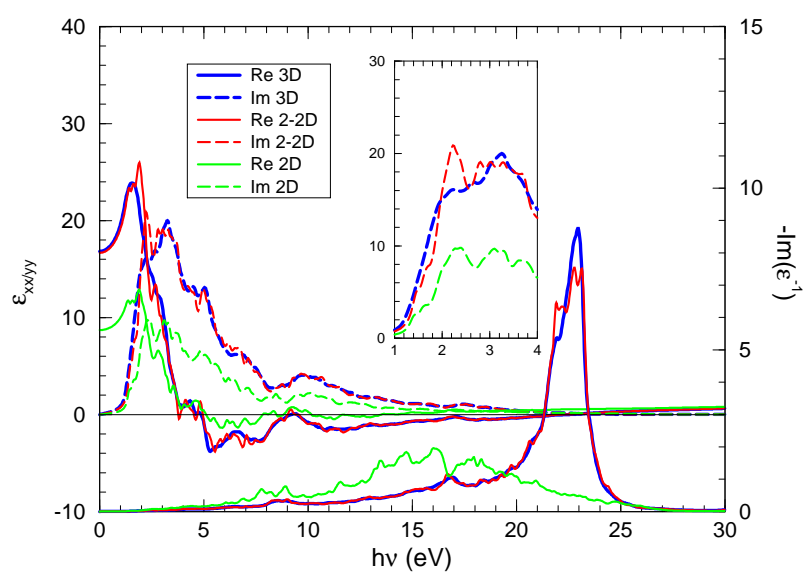

Figura 9: Se muestran los espectros de las componentes reales (líneas continuas) e imaginarias (líneas discontinuas) de la función dieléctrica $\varepsilon_{z z}$ y la función de pérdida $\operatorname{Im}\left(\varepsilon^{-1}\right)$ (líneas continuas) con respecto a la energía de los fotones que abarca desde el infrarrojo hasta el ultravioleta para $\mathrm{WSe}_{2}$, en sus formas 2D (verde), bicapas (rojo) y 3D (azul). En el recuadro se muestra una ampliación de $\varepsilon_{i}$ asociado a la absorción.

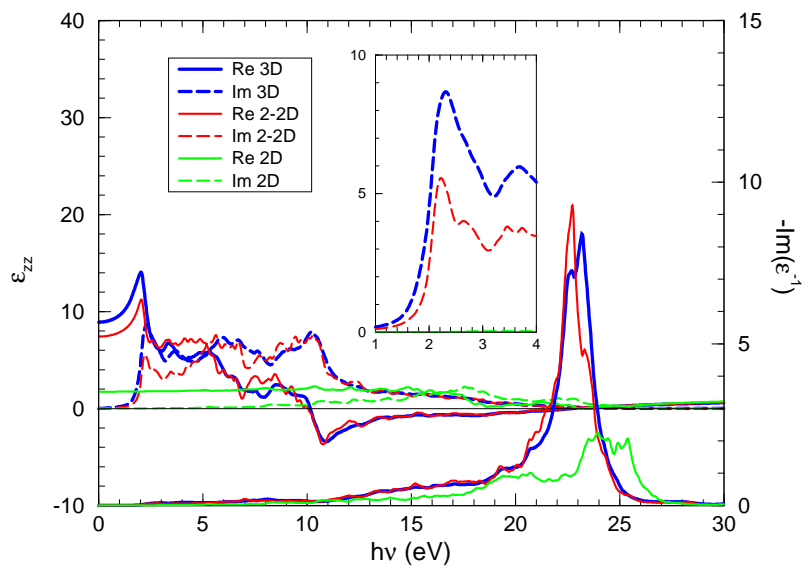

Figura 10: Se muestran los espectros de las componentes reales (líneas continuas) e imaginarias (líneas discontinuas) de la función dieléctrica $\varepsilon_{z z}$ y la función de pérdida $\operatorname{Im}\left(\varepsilon^{-1}\right)$ (líneas continuas) con respecto a la energía de los fotones que abarca desde el infrarrojo hasta el ultravioleta para el $\mathrm{WSe}_{2}$, en sus formas 2D (verde), bicapas (rojo) y 3D (azul). En el recuadro se muestra una ampliación de $\varepsilon_{i}$ asociado a la absorción. 
Para el tercer caso, Figuras 7 y 8 , las componentes imaginarias de $x x$ y $z z$ muestran que los picos de la forma $3 \mathrm{D}$ están en $\approx 2.80,3.10$ y $3.45 \mathrm{eV}$ para el primero, Figura 7 y en $\approx 2.75 \mathrm{eV}$, para el segundo, Figura 8 mientras que para la forma 2-2D están en $\approx 2.33,3.13$ y $3.43 \mathrm{eV}$ para $x x$ y $2.73 \mathrm{eV}$ para $z z$; finalmente para la forma 2D los picos en $x x$ están en $\approx 2.26,3.10$ y 3.30 $\mathrm{eV}$, para $z z$ no existen picos de absorción en la forma 2D.

\begin{tabular}{|c|c|c|c|c|}
\hline \multirow{2}{*}{ Material } & $L$ & $3 \mathrm{D}$ & $2-2 D$ & $2 \mathrm{D}$ \\
\hline & & \multicolumn{3}{|c|}{$\hbar \omega_{p}(\mathrm{eV})$} \\
\hline \multirow{6}{*}{$\mathrm{MoS}_{2}$} & $x x$ & 25.46 & 25.26 & \\
\hline & & & 25.49 & \\
\hline & & & 25.69 & \\
\hline & $\mathrm{zz}$ & 25.66 & 25.24 & \\
\hline & & & 25.54 & \\
\hline & & & 25.76 & \\
\hline \multirow{5}{*}{$\mathrm{MoSe}_{2}$} & $x x$ & 23.41 & 22.91 & \\
\hline & & & 23.66 & \\
\hline & $\mathrm{zz}$ & 23.73 & 23.21 & \\
\hline & & & 23.61 & \\
\hline & & & 24.01 & \\
\hline \multirow{6}{*}{$\mathrm{WS}_{2}$} & $x x$ & 25.69 & 24.19 & \\
\hline & & & 25.54 & \\
\hline & & & 25.79 & \\
\hline & $\mathrm{zz}$ & 25.66 & 25.36 & \\
\hline & & & 25.71 & \\
\hline & & & 26.01 & \\
\hline \multirow{6}{*}{$\mathrm{WSe}_{2}$} & $x x$ & 21.93 & 21.91 & \\
\hline & & 22.96 & 22.18 & \\
\hline & & & 22.78 & \\
\hline & & & 23.13 & \\
\hline & $\mathrm{zz}$ & 22.68 & 22.73 & \\
\hline & & 23.18 & 23.31 & \\
\hline
\end{tabular}

Tabla 2: Se muestran las energías de plasma $\hbar \omega_{p}$ asociados a los valores máximos de la función de pérdida de energía de los electrones que son directamente medibles en Electron Energy Loss Spectroscopy, EELS.

Finalmente, en el cuarto caso que corresponde a $\mathrm{WSe}_{2}$, Figuras 9 y 10, las componentes imaginarias $x x$ presentan los espectros de absorción para las formas 3D, $2-2 D$ y $2 \mathrm{D}$ con una riqueza de picos superpuestos que se torna difícil de identificar sin un detallado diagrama de la estructura electrónica que muestren los diferentes puntos de simetría. Por ejemplo, para la componente $x x$ y la forma 3D se muestran tres picos en forma ascendente de intensidad en $\approx 2.23,2.65$ y $3.25 \mathrm{eV}$, mientras que la forma $2-2 \mathrm{D}$ en intensidades variadas en $\approx 1.46,1.72$, $2.23,2.80,3.03$ y $3.28 \mathrm{eV}$, y la forma $2 \mathrm{D}$ en $\approx 1.44$, $1.69,2.05,2.28,3.10$ y $3.28 \mathrm{eV}$; y para la componente $z z$ la forma 3D tiene un máximo en $2.30 \mathrm{eV}$ y uno menor en $3.68 \mathrm{eV}$, seguido por la forma 2-2D que presenta picos en $\approx 2.23,2.65,3.45$ y $3.73 \mathrm{eV}$, y la componente $z z$ no presenta pico de absorción para la forma 2D.

Por último, en las Figuras 3,10, se muestran para los materiales $\mathrm{MoS}_{2}, \mathrm{MoSe}_{2}, \mathrm{WS}_{2}$ y WSe $\mathrm{W}_{2}$, la formación de plasmones cuando la frecuencia de la radiación electromagnética se hace igual a la frecuencia de oscilación de los electrones -frecuencia de plasma-, que se mueven colectivamente frente a un background de cargas positivas en la banda de valencia. Esta evolución del movimiento colectivo de los electrones se muestra en la gráfica de $-\operatorname{Im}\left(\varepsilon^{-1}\right)$ con líneas de colores azul, rojo y verde para los sistemas 3D, 2-2D y 2D, respectivamente. Cuando las partes imaginarias de $\varepsilon_{x x}, \varepsilon_{y y}$ y $\varepsilon_{z z}$ se aproximan a cero, significando la inexistencia de absorción, la inversa de éstas, la función de pérdida de energía de los electrones, $L=-\operatorname{Im}\left(\varepsilon^{-1}\right)$, alcanzan un máximo valor que se muestran en las respectivas figuras y que se resumen en la tabla 2, notando que las estructuras 2D no poseen frecuencia de plasma significatica en las simulaciones realizadas para los diferentes materiales.

Mencionamos también, que la función de pérdida de energía de los electrones para las estructuras 2-2D presentan hasta tres picos en el caso de $\mathrm{MoS}_{2}$ y WS 2 , y dos picos en el $\mathrm{MoSe}_{2}$, mientras que para el $\mathrm{WSe}_{2}$ presenta cuatro picos en la componente $x x$ y dos picos en la componente $z z$.

\section{Conclusiones}

A través de las partes imaginarias de las funciones dieléctricas hemos observado los espectros de absorción y la formación de plasmones en sistemas 3D, 2-2D y 2D para los dicalcogenuros de metales de transición tales como el $\mathrm{MoS}_{2}, \mathrm{MoSe}_{2}, \mathrm{WS}_{2}$ y WSe $\mathrm{W}_{2}$.

No se forman plasmones entre $25 \mathrm{meV}$ y $30 \mathrm{eV}$ en los sistemas 2D bajo la influencia de un campo electromagnético. Tampoco se observan picos de absorción en la componente zz de los sistemas 2D. Siendo la absorción únicamente producida por el substrato. Estos dos resultados son interesantes en cuanto a que sugieren una técnica para identificar los cristales 2D de los dicalcogenuros de metales de transición. 


\section{Referencias}

[1] G. Onida, L. Reining y A. Rubio, Rev. Mod. Phys. 74, 601 (2001).

[2] F. Aryasetiawan y O. Gunnarsson, Rep. Prog. Phys. 61, 237 (1998). También en arxiv.org condmat/9712013.

[3] E. Runge y E. K. U. Gross, Phys. Rev. Lett. 52, 997 (1984).

[4] Stephan Sagmeister, Excitonic Effects in Solids: Time-Dependent Density Functional Theory versus the Bethe-Salpeter Equation, Dr. Rer. Nat. thesis, Karl-Franzens-Universität Graz, agosto (2009).

[5] E. K. U. Gross y W. Kohn, Phys. Rev. Lett. 55, 2850 (1985).

[6] Peter Y. Yu y Manuel Cardona, Fundamentals of Semiconductors: Physics and Materials Properties, cuarta edición, Springer, Heidelberg (2010).

[7] K. S. Novoselov, D. Jiang, F. Schedin, T. J. Booth, V. V. Khotkevich, S. V. Morozov y A. K. Geim; PNAS 102, 10451 (2005).

[8] Hartmut Haug y Stephan W. Koch, Quantum theory of the optical and electronic properties of semiconductors, tercera edición, World Scientific, Singapore (1994).

[9] Chihiro Hamaguchi, Basic Semiconductors Physics, segunda edición, Springer, Berlin (2010).
[10] John David Jackson, Classical Electrodynamics, tercera edición, John Wiley \& Sons, Inc., Hoboken, NJ (1999).

[11] F. Bruneval, F. Sottile, V. Olevano, R. Del Sole y L. Reining; Phys. Rev. Lett. 94, 186402 (2005).

[12] Yilei Li; Measurement of the Optical Dielectric Function of Monolayer Transition Metal Dichalcogenides: $\mathrm{MoS}_{2}, \mathrm{MoSe}_{2}, \mathrm{WS}_{2}$ y $\mathrm{WSe}_{2}$, cap. 5 del Springer Thesis Probing the Response of Two-Dimensional Crystals by Optical Spectroscopy, Springer, Berlin (2015). Tesis de doctorado, Columbia University presentada en el 2014.

[13] D. I. Arrieta, Y. E. Huamán, M. C. Gutiérrez, R. A. Montalvo y P. H. Rivera; Rev. Inv. Fis. 17, 141701101 (2014).

[14] A. Gulans, S. Kontur, C. Meisenbichler, D. Nabok, P. Pavone, S. Rigamonti, S. Sagmeister, U. Werner y C. Draxl; J. Phys.: Condens. Matter 26, 363202 (2014).

[15] http://exciting-code.org

[16] D. I. Arrieta y P. H. Rivera; Rev. Inv. Fis. 14, 111402751 (2014).

[17] W. Kohn y L. J. Sham; Phys. Rev. 140, A1133 (1964). 